S 9 Table: Cardiac, obstetric, and other indications for the Emergency /Elective lower segment caesarean sections.

\begin{tabular}{|c|c|c|}
\hline Cardiac Indications \pm Obstetric complications & Frequency & Women=Deviation from plan \\
\hline Valve disease with preeclampsia. & $\mathrm{n}=2$ & $\mathrm{n}=2$ \\
\hline Valve disease with decompensated heart failure. & $\mathrm{n}=3$ & $\mathrm{n}=3$ \\
\hline Stable severe stenotic valve disease. & $\mathrm{n}=1$ & $\mathrm{n}=1$ \\
\hline $\begin{array}{l}\text { Dilated cardiomyopathy resulting in severe pulmonary hypertension and / or decompensated heart } \\
\text { failure. }\end{array}$ & $\mathrm{n}=5$ & $\mathrm{n}=5$ \\
\hline Worsening primary pulmonary hypertension with heart failure. & $\mathrm{n}=1$ & $\mathrm{n}=1$ \\
\hline Marfans Syndrome, with significant aortic root dilation. & $\mathrm{n}=3$ & $\mathrm{n}=2$ \\
\hline Type B aortic dissection antepartum. & $\mathrm{n}=1$ & $\mathrm{n}=1$ \\
\hline Complex Cardiovascular Anatomy with preeclampsia. & $\mathrm{n}=1$ & $\mathrm{n}=1$ \\
\hline Cardiac arrests. & $\mathrm{n}=3$ & $\mathrm{n}=3$ \\
\hline Acute pulmonary oedema of unclear cause. & $\mathrm{n}=1$ & $\mathrm{n}=1$ \\
\hline \multicolumn{3}{|l|}{ Obstetric Indications } \\
\hline \multicolumn{3}{|l|}{ Antepartum: } \\
\hline $\begin{array}{l}\text { Placental Abruption ( } n=1) / \text { Placenta Accreta /Placenta Previa, Grade III and IV /Antepartum } \\
\text { haemorrhage. }\end{array}$ & $\mathrm{n}=16$ & $\mathrm{n}=1$ \\
\hline $\begin{array}{l}\text { Pregnancy-induced hypertension (23)/ Preeclampsia and eclampsia (25) with additional obstetric } \\
\text { complications. }\end{array}$ & $\mathrm{n}=48$ & $\mathrm{n}=25$ \\
\hline
\end{tabular}




\section{Delivery related:}

Repeat Lower Segment Caesarean Section (LSCS) +

Failed to progress (FTP)/instrumental delivery or induction of labour + Cord prolapse $(\mathrm{n}=1)$.

Malpresentation: Breech / Brow + cholestasis of pregnancy.

Threatened premature onset labour and /premature onset of labour, with MCDA twins and malpresentation.

Failed instrumental delivery or induction of labour (IOL).

$n=5 \quad n=5$

Premature rupture of membranes $<24$ hours with additional obstetric complications, e.g., threatened

$\mathrm{n}=6$ $\mathrm{n}=50$ premature labour (TPL) with a congenital cardiac condition.

\section{Obstetric Baby related:}

Foetal distress, e.g., Foetal Tachycardia /bradycardia.

$\mathrm{n}=13 \quad \mathrm{n}=13$

Abnormal Doppler $(\mathrm{n}=5) / \mathrm{I} I \mathrm{IUR} /(\mathrm{N}=6) /$ Asymmetrical growth.

$\mathrm{n}=11$

$\mathrm{n}=11$

IUGR + Polyhydramnios/ Oligohydramnios low liquor.

$\mathrm{n}=5$

$\mathrm{n}=5$

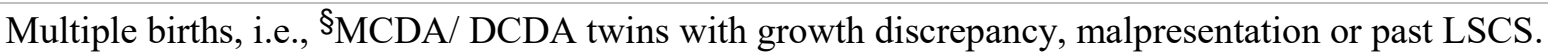

$\mathrm{n}=3$

$\mathrm{n}=3$

\section{Other indications}

Maternal Myotonic Muscular dystrophy (MMD) plus uterine dehiscence.

$\mathrm{n}=1$

I. IVF pregnancy + maternal request, history of pregnancy loss, FTP, repeat LSCS $(n=2)+$

$\mathrm{n}=1$

hypertensive heart disease.

Preexisting poorly controlled hypertension with decompensated heart failure.

Legend, IUGR: intrauterine growth restriction, ${ }^{\S}$ MCDA: monochorionic diamniotic twins share the same chorionic sac but have two amniotic sacs, DCDA: dichorionic diamniotic twins each twin has its own chorionic and amniotic sac, "IVF: in vitro fertilisation. NB: There were women with multiple adverse clinical factors for obstetric indications for LSCS; therefore, overlapping of indications occurred. 